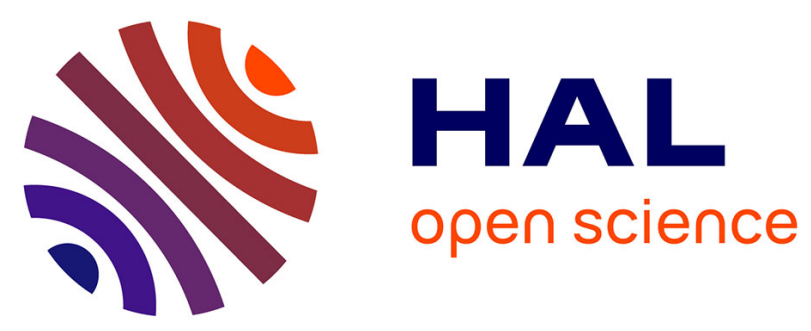

\title{
Convergence of a discretization scheme based on characteristics method for a fluid-rigid system
} Jorge San Martin, Jean-François Scheid, Loredana Smaranda

\section{To cite this version:}

Jorge San Martin, Jean-François Scheid, Loredana Smaranda. Convergence of a discretization scheme based on characteristics method for a fluid-rigid system. Eleventh International Conference on Integral Methods in Science and Engineering (IMSE 2010), Jul 2010, Brighton, United Kingdom. pp.339-348. hal-00589917

\section{HAL Id: hal-00589917 https://hal.science/hal-00589917}

Submitted on 16 May 2017

HAL is a multi-disciplinary open access archive for the deposit and dissemination of scientific research documents, whether they are published or not. The documents may come from teaching and research institutions in France or abroad, or from public or private research centers.
L'archive ouverte pluridisciplinaire HAL, est destinée au dépôt et à la diffusion de documents scientifiques de niveau recherche, publiés ou non, émanant des établissements d'enseignement et de recherche français ou étrangers, des laboratoires publics ou privés. 


\section{Convergence of a discretization scheme based on characteristics method for a fluid-rigid system}

Jorge San Martín*, Jean-François Scheid ${ }^{\S}$, and Loredana Smaranda ${ }^{\ddagger}$

* Universidad de Chile, Santiago, Chile; jorge@dim.uchile.cl

$\S$ Université Henri Poincaré, Nancy, France;

Jean-Francois.Scheid@iecn.u-nancy.fr

$¥$ University of Piteşti, Romania; smaranda@dim.uchile.cl

\subsection{Preliminaries}

In this chapter, we present our last results concerning the convergence of a numerical method to discretize the equations modelling the motion of a rigid solid immersed into a viscous incompressible fluid using the characteristics technique.

Before stating these results, let us introduce the continuous model of our problem. We assume that the fluid-rigid system occupies a bounded and regular domain $\mathcal{O} \subset \mathbb{R}^{2}$ and that the solid is a ball of radius 1 whose center, at time $t$, is denoted by $\boldsymbol{\zeta}(t)$. The fluid fills the part $\Omega(t)=\mathcal{O} \backslash B(\boldsymbol{\zeta}(t))$ at time $t$. The velocity field $\mathbf{u}(\mathbf{x}, t)$ and the pressure $p(\mathbf{x}, t)$ of the fluid, the center of mass $\boldsymbol{\zeta}(t)$ and the angular velocity $\omega(t)$ of the ball satisfy the following Navier-Stokes system coupled with Newton's laws:

$$
\begin{gathered}
\rho_{f}\left(\frac{\partial \mathbf{u}}{\partial t}+(\mathbf{u} \cdot \nabla) \mathbf{u}\right)-\mu \Delta \mathbf{u}+\nabla p=\rho_{f} \mathbf{f}, \mathbf{x} \in \Omega(t), t \in[0, T], \\
\operatorname{div} \mathbf{u}=0, \mathbf{x} \in \Omega(t), t \in[0, T], \\
\mathbf{u}=0, \mathbf{x} \in \partial \mathcal{O}, t \in[0, T], \\
\mathbf{u}=\boldsymbol{\zeta}^{\prime}(t)+\omega(t)(\mathbf{x}-\boldsymbol{\zeta}(t))^{\perp}, \mathbf{x} \in \partial B(\boldsymbol{\zeta}(t)), t \in[0, T], \\
m \boldsymbol{\zeta}^{\prime \prime}(t)=-\int_{\partial B(\boldsymbol{\zeta}(t))} \boldsymbol{\sigma} \mathbf{n} d \Gamma+\rho_{s} \int_{B(\boldsymbol{\zeta}(t))} \mathbf{f}(\mathbf{x}, t) d \mathbf{x}, t \in[0, T], \\
J \omega^{\prime}(t)=-\int_{\partial B(\boldsymbol{\zeta}(t))}(\mathbf{x}-\boldsymbol{\zeta}(t))^{\perp} \cdot \boldsymbol{\sigma} \mathbf{n} d \Gamma+\rho_{s} \int_{B(\boldsymbol{\zeta}(t))}(\mathbf{x}-\boldsymbol{\zeta}(t))^{\perp} \cdot \mathbf{f}(\mathbf{x}, t) d \mathbf{x}, t \in[0, T] .
\end{gathered}
$$

In the above system, $\boldsymbol{\sigma}=-p \mathbf{I} \mathbf{d}+2 \mu \mathrm{D}(\mathbf{u})$ denotes the Cauchy stress tensor with $\mathrm{D}(\mathbf{u})=\left(\nabla \mathbf{u}+\nabla \mathbf{u}^{T}\right) / 2$ and $\nabla \mathbf{u}^{T}$ means the transpose of $\nabla \mathbf{u}$. The positive constant $\mu$ is the dynamic viscosity of the fluid and the constants $m$ and $J$ are the mass and the moment of inertia of the rigid body. Throughout this 
chapter, we will use the notation $\mathbf{x}^{\perp}=\left(\begin{array}{c}-x_{2} \\ x_{1}\end{array}\right)$ for all $\mathbf{x}=\left(\begin{array}{l}x_{1} \\ x_{2}\end{array}\right) \in \mathbb{R}^{2}$. System (1.1)-(1.6) is completed with initial conditions:

$$
\begin{gathered}
\mathbf{u}(\mathbf{x}, 0)=\mathbf{u}_{0}(\mathbf{x}), \quad \mathbf{x} \in \Omega(0), \\
\boldsymbol{\zeta}(0)=\boldsymbol{\zeta}_{0} \in \mathbb{R}^{2}, \quad \boldsymbol{\zeta}^{\prime}(0)=\boldsymbol{\zeta}_{1} \in \mathbb{R}^{2}, \quad \omega(0)=\omega_{0} \in \mathbb{R} .
\end{gathered}
$$

One important hypothesis of our problem is that the density $\rho_{f}$ of the fluid and the density $\rho_{s}$ of the solid are constant, but different, that is

$$
\rho_{f} \neq \rho_{s} .
$$

The fluid-structure interaction problem (1.1)-(1.8) is characterized by the strong coupling between the nonlinear equations of the fluid and those of the structure, as well as the fact that the equations of the fluid are written in a variable domain in time, which depends on the displacement of the structure. Let us now recall some references on the topic. From the numerical point of view, various authors have proposed a number of different techniques to solve equations on moving domains, such as the level set method ([OsSe88]), the fictitious domain method [GPHJP00, GPHJP01]), the immersed boundary method ([Pe02]) and the Arbitrary Lagrangian Eulerian (ALE) method ([MoG197], [Ma99], [FoNo99], [Ga01], [LeTa08], [SMST09]). About numerical convergence for Navier-Stokes equations, when the domain is independent of time, we just recall a few references: [Pi82], [Su88] and [AcGu00]. Concerning the convergence of numerical methods based on finite elements with fixed mesh for a two dimensional fluid-rigid body problem we recall the works [SMSTT04, SMSTT05] where the densities of the fluid and the solid are equal (i.e. $\rho_{f}=\rho_{s}$ ).

The main novelty presented in this chapter is the convergence of two numerical schemes for the generalized case where the densities of the fluid and the solid are not equal (i.e. $\rho_{f} \neq \rho_{s}$ ). The convergence results are given in Theorem 1 and Theorem 2 below, and they concern with the semi-discretization in time variable, respectively the fully-discretization in time and space variables. The complete proofs of these results could be found in our recent papers [SMSS10a, SMSS10b].

Let us now introduce some notation and the functional spaces that we work on. Throughout this chapter, we shall use the classical Sobolev spaces $H^{s}(\mathcal{O}), H_{0}^{s}(\mathcal{O}), H^{-s}(\mathcal{O}), s \geqslant 0$ and the space of Lipschitz continuous functions $C^{0,1}(\overline{\mathcal{O}})$ on the closure of $\mathcal{O}$. We also define

$$
L_{0}^{2}(\mathcal{O})=\left\{f \in L^{2}(\mathcal{O}) \mid \int_{\mathcal{O}} f \mathrm{~d} \mathbf{x}=0\right\} .
$$

The usual inner product in $L^{2}(\mathcal{O})^{2}$ will be denoted by

$$
(\mathbf{u}, \mathbf{v})=\int_{\mathcal{O}} \mathbf{u} \cdot \mathbf{v} \mathrm{d} \mathbf{x} \quad \forall \mathbf{u}, \mathbf{v} \in L^{2}(\mathcal{O})^{2} .
$$


If $\mathbf{A}$ is a matrix, we denote by $\mathbf{A}^{T}$ its transpose. For any $2 \times 2$ matrices $\mathbf{A}, \mathbf{B} \in \mathcal{M}_{2 \times 2}$, we denote by $\mathbf{A}: \mathbf{B}$ their inner product $\mathbf{A}: \mathbf{B}=\operatorname{Trace}\left(\mathbf{A}^{T} \mathbf{B}\right)$, and by $|\mathbf{A}|$ the corresponding norm. For convenience, we use the same notation as in (1.9) for the inner product in $L^{2}\left(\mathcal{O}, \mathcal{M}_{2 \times 2}\right)$, that is

$$
(\mathbf{A}, \mathbf{B})=\int_{\mathcal{O}} \mathbf{A}: \mathbf{B} \mathrm{d} \mathbf{x} \quad \forall \mathbf{A}, \mathbf{B} \in L^{2}\left(\mathcal{O}, \mathcal{M}_{2 \times 2}\right) .
$$

For $\boldsymbol{\zeta} \in \mathcal{O}$, we introduce the space of rigid functions in $B(\boldsymbol{\zeta})=\left\{\mathrm{x} \in \mathbb{R}^{2}\right.$ : $|\mathbf{x}-\zeta| \leq 1\}$,

$$
\mathcal{K}(\boldsymbol{\zeta})=\left\{\mathbf{u} \in H_{0}^{1}(\mathcal{O})^{2} \mid \mathrm{D}(\mathbf{u})=0 \text { in } B(\boldsymbol{\zeta})\right\},
$$

the space of rigid functions in $B(\boldsymbol{\zeta})$ with divergence free in the whole domain $\mathcal{O}$,

$$
\widehat{\mathcal{K}}(\boldsymbol{\zeta})=\{\mathbf{u} \in \mathcal{K}(\boldsymbol{\zeta}) \mid \operatorname{div} \mathbf{u}=0 \text { in } \mathcal{O}\}
$$

and the space of the pressure

$$
M(\boldsymbol{\zeta})=\left\{p \in L_{0}^{2}(\mathcal{O}) \mid p=0 \text { in } B(\boldsymbol{\zeta})\right\} .
$$

Let us consider in the reminder of the chapter that any velocity field in $\mathcal{K}(\boldsymbol{\zeta})$ will be extended by zero outside of $\mathcal{O}$.

According to Lemma 1.1 of [Te83, pp.18], for any $\mathbf{u} \in \mathcal{K}(\boldsymbol{\zeta})$, there exist $\mathbf{l}_{u} \in \mathbb{R}^{2}$ and $\omega_{\mathbf{u}} \in \mathbb{R}$ such that

$$
\mathbf{u}(\mathbf{y})=\mathbf{l}_{\mathbf{u}}+\omega_{\mathbf{u}}(\mathbf{y}-\boldsymbol{\zeta})^{\perp} \quad \forall \mathbf{y} \in B(\boldsymbol{\zeta}) .
$$

In addition, we define the density $\rho$ by the following piecewise constant function

$$
\rho(\mathbf{x})=\left\{\begin{array}{l}
\rho_{s} \text { if } \mathbf{x} \in B(\boldsymbol{\zeta}), \\
\rho_{f} \text { if } \mathbf{x} \in \mathcal{O} \backslash B(\boldsymbol{\zeta}) .
\end{array}\right.
$$

We notice that, by using the above definitions, for any $\mathbf{u}, \mathbf{v} \in \mathcal{K}(\boldsymbol{\zeta})$ we have

$$
(\rho \mathbf{u}, \mathbf{v})=\int_{\mathcal{O} \backslash B(\boldsymbol{\zeta})} \rho_{f} \mathbf{u} \cdot \mathbf{v} \mathrm{d} \mathbf{x}+M \mathbf{l}_{\mathbf{u}} \cdot \mathbf{l}_{\mathbf{v}}+J \omega_{\mathbf{u}} \omega_{\mathbf{v}}
$$

The spaces (1.10)-(1.11) are specific to our problem. In fact, if the solution $\mathbf{u}$ of (1.1)-(1.8) is extended by

$$
\mathbf{u}(\mathbf{x}, t)=\boldsymbol{\zeta}^{\prime}(t)+\omega(t)(\mathbf{x}-\boldsymbol{\zeta}(t))^{\perp} \quad \forall \mathbf{x} \in B(\boldsymbol{\zeta}(t)),
$$

then, we easily see that $\mathbf{u}(t) \in \widehat{\mathcal{K}}(\boldsymbol{\zeta}(t))$. In the reminder of this chapter, the solution $\mathbf{u}$ of (1.1)-(1.8) will be extended as above.

An important ingredient of the numerical method we use is given by the characteristic functions whose level lines are the integral curves of the velocity field. More precisely (see, for instance, [Pi82], [Su88]) the characteristic 
function $\widetilde{\psi}:[0, T]^{2} \times \mathcal{O} \rightarrow \mathcal{O}$ is defined as the solution of the initial value problem

$$
\left\{\begin{array}{l}
\frac{d}{d t} \widetilde{\boldsymbol{\psi}}(t ; s, \mathbf{x})=\mathbf{u}(\widetilde{\boldsymbol{\psi}}(t ; s, \mathbf{x}), t) \quad \forall t \in[0, T], \\
\widetilde{\boldsymbol{\psi}}(s ; s, \mathbf{x})=\mathbf{x} .
\end{array}\right.
$$

It is well-known that the material derivative $D_{t} \mathbf{u}=\partial \mathbf{u} / \partial t+(\mathbf{u} \cdot \nabla) \mathbf{u}$ of $\mathbf{u}$ at instant $t_{0}$ satisfies:

$$
D_{t} \mathbf{u}\left(\mathbf{x}, t_{0}\right)=\frac{d}{d t}\left[\mathbf{u}\left(\widetilde{\boldsymbol{\psi}}\left(t ; t_{0}, \mathbf{x}\right), t\right)\right]_{\left.\right|_{t=t_{0}}} .
$$

Remark 1. By using a classical result of Liouville (see, for instance, [Ar92, pp.251]), if

$$
\boldsymbol{\zeta} \in H^{2}(0, T)^{2}, \quad \omega \in H^{1}(0, T), \quad \mathbf{u} \in C([0, T] ; \widehat{\mathcal{K}}(\boldsymbol{\zeta}(t))),
$$

then we have that

$$
\operatorname{det} \mathbf{J}_{\widetilde{\psi}}=1
$$

where we have denoted by $\mathbf{J}_{\widetilde{\psi}}=\left(\frac{\partial \widetilde{\psi}_{i}}{\partial y_{j}}\right)_{i, j}$ the jacobian matrix of the transformation $\mathbf{y} \mapsto \widetilde{\boldsymbol{\psi}}(\mathbf{y})$.

Let us now state the weak formulation of the system (1.1)-(1.8) that we use to discretize in time the problem.

Proposition 1. Assume that

$$
\begin{gathered}
\mathbf{u} \in L^{2}\left(0, T ; H^{2}(\Omega(t))^{2}\right) \cap H^{1}\left(0, T ; L^{2}(\Omega(t))^{2}\right) \cap C\left([0, T] ; H^{1}(\Omega(t))^{2}\right), \\
p \in L^{2}\left(0, T ; H^{1}(\Omega(t))\right), \quad \zeta \in H^{2}(0, T)^{2}, \quad \omega \in H^{1}(0, T)
\end{gathered}
$$

and that $\mathbf{u}$ is extended by

$$
\mathbf{u}(\mathbf{x}, t)=\boldsymbol{\zeta}^{\prime}(t)+\omega(t)(\mathbf{x}-\boldsymbol{\zeta}(t))^{\perp} \quad \forall \mathbf{x} \in B(\boldsymbol{\zeta}(t)) .
$$

Then $(\mathbf{u}, p, \boldsymbol{\zeta}, \omega)$ is the solution of (1.1)-(1.8) if and only if for all $t \in[0, T]$, $\mathbf{u}(\cdot, t) \in \mathcal{K}(\boldsymbol{\zeta}(t)), p(\cdot, t) \in M(\boldsymbol{\zeta}(t))$ and $(\mathbf{u}, p)$ satisfies

$$
\begin{gathered}
\left(\rho \frac{d}{d t}[\mathbf{u} \circ \tilde{\boldsymbol{\psi}}](t), \boldsymbol{\varphi}\right)+a(\mathbf{u}, \boldsymbol{\varphi})+b(\boldsymbol{\varphi}, p)=(\rho \mathbf{f}(t), \boldsymbol{\varphi}) \quad \forall \boldsymbol{\varphi} \in \mathcal{K}(\boldsymbol{\zeta}(t)), \\
b(\mathbf{u}, q)=0 \quad \forall q \in M(\boldsymbol{\zeta}(t)),
\end{gathered}
$$

where the bilinear forms $a(\cdot, \cdot)$ and $b(\cdot, \cdot)$ are defined as follows:

$$
a(\mathbf{u}, \mathbf{v})=2 \mu \int_{\mathcal{O}} \mathrm{D}(\mathbf{u}): \mathrm{D}(\mathbf{v}) \mathrm{d} \mathbf{x} \quad \forall \mathbf{u}, \mathbf{v} \in H^{1}(\mathcal{O})^{2}
$$

and

$$
b(\mathbf{u}, p)=-\int_{\mathcal{O}} \operatorname{div}(\mathbf{u}) p \mathrm{~d} \mathbf{x} \quad \forall \mathbf{u} \in H^{1}(\mathcal{O})^{2}, \forall p \in L_{0}^{2}(\mathcal{O}) .
$$


For the proof of Proposition 1 we refer the reader to [QuVa94, Ch.12]. In the remainder of this chapter, we suppose that $\mathbf{f}$ and $\mathbf{u}_{0}$ satisfy

$$
\begin{aligned}
& \mathbf{f} \in C\left([0, T] ; H^{1}(\mathcal{O})^{2}\right), \quad \mathbf{u}_{0} \in H^{2}(\Omega)^{2}, \quad \operatorname{div}\left(\mathbf{u}_{0}\right)=0 \quad \text { in } \Omega, \\
& \mathbf{u}_{0}=0 \quad \text { on } \partial \mathcal{O}, \quad \mathbf{u}_{0}(\mathbf{y})=\boldsymbol{\zeta}_{1}+\omega_{0}\left(\mathbf{y}-\boldsymbol{\zeta}_{0}\right)^{\perp} \quad \text { on } \partial B\left(\boldsymbol{\zeta}_{0}\right),
\end{aligned}
$$

where $\boldsymbol{\zeta}_{0}, \boldsymbol{\zeta}_{1} \in \mathbb{R}^{2}, \omega_{0} \in \mathbb{R}$ and $\Omega=\mathcal{O} \backslash B\left(\boldsymbol{\zeta}_{0}\right)$. Let us also assume that the corresponding solution $(\mathbf{u}, p, \boldsymbol{\zeta}, \omega)$ of problem (1.1)-(1.8) satisfies

$$
\left\{\begin{array}{l}
\mathbf{u} \in C\left([0, T] ; H^{2}(\Omega(t))^{2}\right) \cap H^{1}\left(0, T ; L^{2}(\Omega(t))^{2}\right), \\
D_{t}^{2} \mathbf{u} \in L^{2}\left(0, T ; L^{2}(\Omega(t))^{2}\right), \quad \mathbf{u} \in C\left([0, T] ; C^{0,1}(\overline{\mathcal{O}})^{2}\right) \\
p \in C\left([0, T] ; H^{1}(\Omega(t))\right), \quad \zeta \in H^{3}(0, T)^{2}, \quad \omega \in H^{2}(0, T)
\end{array}\right.
$$

and

$$
\operatorname{dist}(B(\boldsymbol{\zeta}(t)), \partial \mathcal{O})>0 \quad \forall t \in[0, T] .
$$

Remark 2. The hypotheses (1.23) and (1.24) imply the existence of $\eta>0$ such that

$$
\operatorname{dist}(B(\boldsymbol{\zeta}(t)), \partial \mathcal{O})>3 \eta \quad \forall t \in[0, T] .
$$

\subsection{Semi-discretization in time variable}

By using the weak formulation (1.18)-(1.19), let us derive a semi-discrete version of our system. For $N \in \mathbb{N}^{*}$ we denote $\Delta t=T / N$ and $t_{k}=k \Delta t$ for $k=0, \ldots, N$. Denote by $\left(\mathbf{u}^{k}, \boldsymbol{\zeta}^{k}\right) \in \widehat{\mathcal{K}}\left(\boldsymbol{\zeta}^{k}\right) \cap C^{0}(\overline{\mathcal{O}})^{2} \times \mathcal{O}$ the approximation of the solution of (1.1)-(1.8) at the time $t=t_{k}$. In the sequel, we shall use the notation

$$
\widetilde{\mathbf{X}}(\mathbf{x})=\widetilde{\boldsymbol{\psi}}\left(t_{k} ; t_{k+1}, \mathbf{x}\right) \quad \forall \mathbf{x} \in \mathcal{O} .
$$

We approximate the position of the rigid ball at instant $t_{k+1}$ by $\boldsymbol{\zeta}^{k+1}$ which is defined by the relation

$$
\zeta^{k+1}=\zeta^{k}+\mathbf{u}^{k}\left(\zeta^{k}\right) \Delta t
$$

We then define the characteristic function $\bar{\psi}$ associated with the semidiscretized velocity field as the solution of

$$
\left\{\begin{array}{l}
\frac{d}{d t} \overline{\boldsymbol{\psi}}\left(t ; t_{k+1}, \mathbf{x}\right)=\mathbf{u}^{k}\left(\overline{\boldsymbol{\psi}}\left(t ; t_{k+1}, \mathbf{x}\right)\right)-\mathbf{u}^{k}\left(\boldsymbol{\zeta}^{k}\right) \quad \forall t \in\left[t_{k}, t_{k+1}\right] \\
\overline{\boldsymbol{\psi}}\left(t_{k+1} ; t_{k+1}, \mathbf{x}\right)=\mathbf{x}-\mathbf{u}^{k}\left(\boldsymbol{\zeta}^{k}\right) \Delta t
\end{array}\right.
$$

and we denote

$$
\overline{\mathbf{X}}^{k}(\mathbf{x})=\overline{\boldsymbol{\psi}}\left(t_{k} ; t_{k+1}, \mathbf{x}\right) \quad \forall \mathbf{x} \in \mathcal{O} .
$$

In equation (1.28), the velocity field $\mathbf{u}^{k}$ is extended by zero outside of the domain $\mathcal{O}$. 
We next define $\mathbf{u}^{k+1} \in \widehat{\mathcal{K}}\left(\boldsymbol{\zeta}^{k+1}\right)$ as the solution of the following Stokes type system

$$
\begin{aligned}
\left(\rho^{k+1} \frac{\mathbf{u}^{k+1}-\mathbf{u}^{k} \circ \overline{\mathbf{X}}^{k}}{\Delta t}, \boldsymbol{\varphi}\right)+a\left(\mathbf{u}^{k+1}, \boldsymbol{\varphi}\right) & \\
& =\left(\rho^{k+1} \mathbf{f}^{k+1}, \boldsymbol{\varphi}\right) \quad \forall \boldsymbol{\varphi} \in \widehat{\mathcal{K}}\left(\boldsymbol{\zeta}^{k+1}\right),
\end{aligned}
$$

where $\mathbf{f}^{k+1}=\mathbf{f}\left(t_{k+1}\right)$ and $\rho^{k+1}$ is defined by

$$
\rho^{k+1}(\mathbf{x})=\left\{\begin{array}{l}
\rho_{s} \text { if } \mathbf{x} \in B\left(\boldsymbol{\zeta}^{k+1}\right), \\
\rho_{f} \text { if } \mathbf{x} \in \mathcal{O} \backslash B\left(\boldsymbol{\zeta}^{k+1}\right) .
\end{array}\right.
$$

The above equation can be rewritten by using a mixed formulation. It is clear that (1.30) is equivalent to the following system

$$
\begin{gathered}
\left(\rho^{k+1} \frac{\mathbf{u}^{k+1}-\mathbf{u}^{k} \circ \overline{\mathbf{X}}^{k}}{\Delta t}, \boldsymbol{\varphi}\right)+a\left(\mathbf{u}^{k+1}, \boldsymbol{\varphi}\right)+b\left(\boldsymbol{\varphi}, p^{k+1}\right) \\
=\left(\rho^{k+1} \mathbf{f}^{k+1}, \boldsymbol{\varphi}\right) \quad \forall \boldsymbol{\varphi} \in \mathcal{K}\left(\boldsymbol{\zeta}^{k+1}\right), \\
b\left(\mathbf{u}^{k+1}, q\right)=0 \quad \forall q \in M\left(\boldsymbol{\zeta}^{k+1}\right),
\end{gathered}
$$

of unknowns $\left(\mathbf{u}^{k+1}, p^{k+1}\right) \in \mathcal{K}\left(\boldsymbol{\zeta}^{k+1}\right) \times M\left(\boldsymbol{\zeta}^{k+1}\right)$.

It is well-known (see, for example, [GiRa79, Corollary I.4.1., pp.61]) that the mixed formulation (1.31)-(1.32) is a well-posed problem, provided that the spaces $\mathcal{K}(\boldsymbol{\zeta}), M(\boldsymbol{\zeta})$ and the bilinear form $b$ satisfy an inf-sup condition. The fact that this inf-sup condition is satisfied in our case follows from the result below (for the proof see, for instance [GiRa79, pp.81]):

Lemma 1. Suppose that $\boldsymbol{\zeta} \in \mathcal{O}$ is such that $d(\boldsymbol{\zeta}, \partial \mathcal{O})=1+\eta$, with $\eta>0$. Then there exists a constant $\beta>0$, depending only on $\eta$ and on $\mathcal{O}$, such that for all $q \in M(\boldsymbol{\zeta})$ there exists $\mathbf{u} \in \mathcal{K}(\boldsymbol{\zeta})$ with

$$
\int_{\mathcal{O}} \operatorname{div}(\mathbf{u}) q \mathrm{~d} \mathbf{x} \geq \beta\|\mathbf{u}\|_{H^{1}(\mathcal{O})^{2}}\|q\|_{L^{2}(\mathcal{O})} .
$$

In addition, we have that $\mathbf{u}^{k+1} \in C^{0}(\overline{\mathcal{O}})^{2}$ (for more details, see [SMSS10b]).

Let us now state the first main result concerning the convergence of the semi-discrete scheme (1.31)-(1.32) (for the proof of the next theorem, we refer the reader to [SMSS10b]):

Theorem 1. Suppose that $\mathcal{O}$ is an open smooth bounded domain in $\mathbb{R}^{2}, \mathbf{f}$ and $\mathbf{u}_{0}$ satisfy (1.22) and $(\mathbf{u}, p, \boldsymbol{\zeta}, \omega)$ is a solution of (1.1)-(1.8) satisfying $(1.23)-(1.24)$.

Then there exist two positive constants $C$ and $\tau^{*}$ not depending on $\Delta t$ such that for all $0<\Delta t \leqslant \tau^{*}$ the solution $\left(\mathbf{u}^{k}, p^{k}, \boldsymbol{\zeta}^{k}\right)$ of the semi-discretization problem (1.31)-(1.32) satisfies

$$
\sup _{1 \leqslant k \leqslant N}\left(\left|\boldsymbol{\zeta}\left(t_{k}\right)-\boldsymbol{\zeta}^{k}\right|+\left\|\mathbf{u}\left(t_{k}\right)-\mathbf{u}^{k}\right\|_{L^{2}(\mathcal{O})^{2}}\right) \leqslant C \Delta t .
$$


The key ingredients used in the proof of the above result are some properties on the characteristic functions associated with the semi-discretized velocity field which are given in the following lemma (more details and the complete proof of this result could be found in [SMSS10b]):

Lemma 2. For any $k \in\{0, \ldots, N\}$, the characteristic function $\overline{\boldsymbol{\psi}}$ defined in (1.28)-(1.29) satisfies the following properties:

i) $\overline{\mathbf{X}}^{k}\left(B\left(\boldsymbol{\zeta}^{k+1}\right)\right)=B\left(\boldsymbol{\zeta}^{k}\right)$;

ii) If we extend by $\rho_{f}$ the density field $\rho^{k}$ outside of $\mathcal{O}$, we have

$$
\rho^{k+1}=\rho^{k} \circ \overline{\mathbf{X}}^{k}
$$

iii) For any $f \in L^{2}\left(\mathbb{R}^{2}\right)$ such that $f=0$ in $\mathbb{R}^{2} \backslash \mathcal{O}$, we have

$$
\left\|f \circ \overline{\boldsymbol{\psi}}\left(t ; t_{k+1}, \cdot\right)\right\|_{L^{2}(\mathcal{O})} \leq\|f\|_{L^{2}(\mathcal{O})} \quad \forall t \in\left[t_{k}, t_{k+1}\right] .
$$

\subsection{Fully discretization in time and space variables}

In order to discretize the problem (1.31)-(1.32) with respect to the space variable, let us introduce two families of finite element spaces which approximate the spaces $\mathcal{K}(\boldsymbol{\zeta})$ and $M(\boldsymbol{\zeta})$ defined in (1.10) and (1.12). To this end, we consider the discretization parameter $0<h<1$.

Let $\mathcal{T}_{h}$ be a quasi-uniform triangulation of the domain $\mathcal{O}$. We denote by $\mathcal{W}_{h}$ the $\mathbb{P}_{1}$-bubble finite elements space associated with $\mathcal{T}_{h}$ for the velocity field in the Stokes problem and by $E_{h}$ the $\mathbb{P}_{1}$-finite elements space for the pressure. Then, we define the following finite elements spaces for a conform approximation of the fluid-rigid system:

$$
\begin{aligned}
\mathcal{K}_{h}(\boldsymbol{\zeta}) & =\mathcal{W}_{h} \cap \mathcal{K}(\boldsymbol{\zeta}) & & \forall \boldsymbol{\zeta} \in \mathcal{O} \\
M_{h}(\boldsymbol{\zeta}) & =E_{h} \cap M(\boldsymbol{\zeta}) & & \forall \boldsymbol{\zeta} \in \mathcal{O} .
\end{aligned}
$$

In order to define the approximate characteristics, let us denote by $F_{h}$ the $\mathbb{P}_{2}$-finite element space associated with the triangulation $\mathcal{T}_{h}$ and we introduce the space:

$$
\mathcal{R}_{h}(\boldsymbol{\zeta})=\left\{\nabla^{\perp} \varphi_{h}: \varphi_{h} \in F_{h}, \varphi_{h}=0 \text { on } \partial \mathcal{O}\right\} \cap \mathcal{K}(\boldsymbol{\zeta}) \quad \forall \boldsymbol{\zeta} \in \mathcal{O},
$$

where we have denoted by $\nabla^{\perp} \varphi_{h}=\left(\begin{array}{c}-\frac{\partial \varphi_{h}}{\partial y} \\ \frac{\partial \varphi_{h}}{\partial x}\end{array}\right)$.

We denote $\mathbf{P}(\boldsymbol{\zeta})$ the orthogonal projection from $L^{2}(\mathcal{O})^{2}$ onto $\mathcal{R}_{h}(\boldsymbol{\zeta})$, i.e. if $\mathbf{u} \in L^{2}(\mathcal{O})^{2}$ then $\mathbf{P}(\boldsymbol{\zeta}) \mathbf{u} \in \mathcal{R}_{h}(\boldsymbol{\zeta})$ such that $\left(\mathbf{u}-\mathbf{P}(\boldsymbol{\zeta}) \mathbf{u}, \mathbf{r}_{h}\right)=0$ for all $\mathbf{r}_{h} \in \mathcal{R}_{h}(\boldsymbol{\zeta})$. 
Let $N$ be a positive integer. We denote $\Delta t=T / N$ and $t_{k}=k \Delta t$ for all $k \in\{0, \ldots, N\}$. Assume that the approximate solution $\left(\mathbf{u}_{h}^{k}, p_{h}^{k}, \boldsymbol{\zeta}_{h}^{k}\right)$ of (1.1)(1.8) at $t=t_{k}$ is known. We describe below the numerical scheme allowing to determinate the approximate solution $\left(\mathbf{u}_{h}^{k+1}, p_{h}^{k+1}, \boldsymbol{\zeta}_{h}^{k+1}\right)$ at $t=t_{k+1}$. First, we compute $\boldsymbol{\zeta}_{h}^{k+1} \in \mathbb{R}^{2}$ by

$$
\boldsymbol{\zeta}_{h}^{k+1}=\boldsymbol{\zeta}_{h}^{k}+\mathbf{u}_{h}^{k}\left(\boldsymbol{\zeta}_{h}^{k}\right) \Delta t
$$

We consider the approximated characteristic function $\overline{\boldsymbol{\psi}}_{h}^{k}$ defined as the solution of

$$
\left\{\begin{array}{l}
\frac{d}{d t} \overline{\boldsymbol{\psi}}_{h}^{k}\left(t ; t_{k+1}, \mathbf{x}\right)=\mathbf{P}\left(\boldsymbol{\zeta}_{h}^{k}\right) \mathbf{u}_{h}^{k}\left(\overline{\boldsymbol{\psi}}_{h}^{k}\left(t ; t_{k+1}, \mathbf{x}\right)\right)-\mathbf{P}\left(\boldsymbol{\zeta}_{h}^{k}\right) \mathbf{u}_{h}^{k}\left(\boldsymbol{\zeta}_{h}^{k}\right) \quad \forall t \in\left[t_{k}, t_{k+1}\right] \\
\overline{\boldsymbol{\psi}}_{h}^{k}\left(t_{k+1} ; t_{k+1}, \mathbf{x}\right)=\mathbf{x}-\mathbf{u}_{h}^{k}\left(\boldsymbol{\zeta}_{h}^{k}\right) \Delta t
\end{array}\right.
$$

and we define

$$
\overline{\mathbf{X}}_{h}^{k}(\mathbf{x})=\overline{\boldsymbol{\psi}}_{h}^{k}\left(t_{k} ; t_{k+1}, \mathbf{x}\right) \quad \forall \mathbf{x} \in \mathcal{O} .
$$

We observe that since $\operatorname{div}\left(\mathbf{P}\left(\boldsymbol{\zeta}_{h}^{k}\right) \mathbf{u}_{h}^{k}\left(\overline{\boldsymbol{\psi}}_{h}^{k}\left(t ; t_{k+1}, \cdot\right)\right)-\mathbf{P}\left(\boldsymbol{\zeta}_{h}^{k}\right) \mathbf{u}_{h}^{k}\left(\boldsymbol{\zeta}_{h}^{k}\right)\right)=0$ and $\nabla\left(\mathbf{x}-\mathbf{u}_{h}^{k}\left(\boldsymbol{\zeta}_{h}^{k}\right) \Delta t\right)=\mathbf{I d}$, we get

$$
\operatorname{det} \mathbf{J}_{\bar{\psi}_{h}^{k}}=1 .
$$

Let us split the mesh into the union of 4 different types of triangle's subsets. We first introduce $A_{h}$ as the union of all triangles intersecting the ball $B\left(\boldsymbol{\zeta}_{h}^{k}\right)$, i.e.

$$
\begin{gathered}
A_{h}=\underset{\substack{T \in \mathcal{T}_{h} \\
\stackrel{\circ}{T} \cap \stackrel{\leftrightarrow}{B}\left(\boldsymbol{\zeta}_{h}^{k}\right) \neq \emptyset}}{\bigcup} T .
\end{gathered}
$$

We also denote by $Q_{h}$ the union of all triangles such that all their vertices are contained in $\overline{A_{h}}$. The triangles of $\mathcal{T}_{h}$ are then splitted into the 4 following categories:

- $\quad \mathcal{F}_{1}$ is the subset of $\mathcal{T}_{h}$ formed by all triangles $T \in \mathcal{T}_{h}$ such that $\bar{T} \subset B\left(\boldsymbol{\zeta}_{h}^{k}\right)$.

- $\mathcal{F}_{2}$ is the subset formed by all triangles $T \in \mathcal{T}_{h} \backslash \mathcal{F}_{1}$ such that $\bar{T} \subset \overline{Q_{h}}$.

- $\mathcal{F}_{3}$ is the subset formed by all triangles $T \in \mathcal{T}_{h}$ such that $\bar{T} \cap \overline{Q_{h}} \neq \emptyset$ and $T \not \subset \overline{Q_{h}}$.

- $\mathcal{F}_{4}=\mathcal{T}_{h} \backslash\left(\mathcal{F}_{1} \cup \mathcal{F}_{2} \cup \mathcal{F}_{3}\right)$.

We introduce two approximated density functions $\rho_{h}^{k}$ and $\bar{\rho}_{h}^{k}$ as follows:

$$
\rho_{h}^{k}(\mathbf{x})=\left\{\begin{array}{l}
\rho_{s} \text { if } \mathbf{x} \in B\left(\boldsymbol{\zeta}_{h}^{k}\right) \\
\rho_{f} \text { if } \mathbf{x} \in \mathcal{O} \backslash B\left(\boldsymbol{\zeta}_{h}^{k}\right)
\end{array}\right.
$$

and 


$$
\bar{\rho}_{h}^{k}(\mathbf{x})=\left\{\begin{array}{l}
\rho_{s} \text { if } \mathbf{x} \in \overline{Q_{h}}, \\
\rho_{f} \text { if } \mathbf{x} \in \mathcal{F}_{4} .
\end{array}\right.
$$

With these notations, we consider the following mixed variational fully discrete formulation: Find $\left(\mathbf{u}_{h}^{k+1}, p_{h}^{k+1}\right) \in \mathcal{K}_{h}\left(\boldsymbol{\zeta}_{h}^{k+1}\right) \times M_{h}\left(\boldsymbol{\zeta}_{h}^{k+1}\right)$ such that

$$
\begin{aligned}
\left(\rho_{h}^{k+1} \frac{\mathbf{u}_{h}^{k+1}-\mathbf{u}_{h}^{k} \circ \overline{\mathbf{X}}_{h}^{k}}{\Delta t}, \boldsymbol{\varphi}\right)+ & a\left(\mathbf{u}_{h}^{k+1}, \boldsymbol{\varphi}\right)+b\left(\boldsymbol{\varphi}, p_{h}^{k+1}\right) \\
= & \left(\bar{\rho}_{h}^{k+1} \mathbf{f}_{h}^{k+1}, \boldsymbol{\varphi}\right) \quad \forall \boldsymbol{\varphi} \in \mathcal{K}_{h}\left(\boldsymbol{\zeta}_{h}^{k+1}\right) \\
b\left(\mathbf{u}_{h}^{k+1}, q\right) & =0 \quad \forall q \in M_{h}\left(\boldsymbol{\zeta}_{h}^{k+1}\right)
\end{aligned}
$$

where $\mathbf{f}_{h}^{k+1}$ is the $L^{2}(\mathcal{O})^{2}$-projection of $\mathbf{f}^{k+1}=\mathbf{f}\left(t_{k+1}\right)$ on $\left(E_{h}\right)^{2}$.

Let us now state the second result of this chapter concerning the convergence of the fully-discrete scheme (1.42)-(1.43) (for the proof of this result, we refer the reader to [SMSS10b]):

Theorem 2. Let $\mathcal{O}$ be a convex domain with a polygonal boundary. Suppose that $\mathbf{f}$ and $\mathbf{u}_{0}$ satisfy the conditions (1.22) and that $(\mathbf{u}, p, \boldsymbol{\zeta}, \omega)$ is a solution of (1.1)-(1.8) satisfying the regularity properties (1.23) and such that (1.24) holds. Let $C_{0}>0$ be a fixed constant.

Then there exist two positive constants $C$ and $\tau^{*}$ independent of $h$ and $\Delta t$ such that for all $0<\Delta t \leq \tau^{*}$ and for all $h \leq C_{0} \Delta t^{2}$ we have

$$
\sup _{1 \leq k \leq N}\left(\left|\boldsymbol{\zeta}\left(t_{k}\right)-\boldsymbol{\zeta}_{h}^{k}\right|+\left\|\mathbf{u}\left(t_{k}\right)-\mathbf{u}_{h}^{k}\right\|_{L^{2}(\mathcal{O})^{2}}\right) \leq C \Delta t .
$$

The key ingredients in the proof of the previous convergence result are the properties on the characteristic functions associated with the fully-discretized velocity field given in the following lemma (the proof of this result is analogous to the proof of Lemma 2 and could be found in [SMSS10b]):

Lemma 3. For any $k \in\{0, \ldots, N\}$ and $h \in(0,1)$, the characteristic function $\overline{\boldsymbol{\psi}}_{h}^{k}$ defined in (1.37)-(1.38) satisfies the following properties:

i) $\overline{\mathbf{X}}_{h}^{k}\left(B\left(\boldsymbol{\zeta}_{h}^{k+1}\right)\right)=B\left(\boldsymbol{\zeta}_{h}^{k}\right)$;

ii) If we extend by $\rho_{f}$ the density field $\rho_{h}^{k}$ outside of $\mathcal{O}$, we have

$$
\rho_{h}^{k+1}=\rho_{h}^{k} \circ \overline{\mathbf{X}}_{h}^{k} ;
$$

iii) For any $f \in L^{2}\left(\mathbb{R}^{2}\right)$ such that $f=0$ in $\mathbb{R}^{2} \backslash \mathcal{O}$, we have

$$
\left\|f \circ \overline{\boldsymbol{\psi}}_{h}^{k}\left(t ; t_{k+1}, \cdot\right)\right\|_{L^{2}(\mathcal{O})^{2}} \leq\|f\|_{L^{2}(\mathcal{O})^{2}} \quad \forall t \in\left[t_{k}, t_{k+1}\right] .
$$




\section{Acknowledgements}

J. San Martín was partially supported by Grant Fondecyt 1090239 and BASAL-CMM Project. J.-F. Scheid gratefully acknowledges the Program ECOS-CONICYT (Scientific cooperation project between France and Chile) through grant C07-E05. L. Smaranda was partially supported by Grant RP-2, no. 6/01.07.2009 of CNCSIS-UEFISCSU.

\section{References}

[AcGu00] Achdou, Y., Guermond, J.-L.: Convergence analysis of a finite element projection/Lagrange-Galerkin method for the incompressible NavierStokes equations. SIAM J. Numer. Anal., 37, 799-826 (2000).

[Ar92] Arnold, V.: Ordinary differential equations. Springer-Verlag, Berlin (1992).

[FoNo99] Formaggia, L., Nobile, F.: A stability analysis for the arbitrary Lagrangian Eulerian formulation with finite elements. East-West J. Numer. Math., 7, 105-131 (1999).

[Ga01] Gastaldi, L.: A priori error estimates for the arbitrary Lagrangian Eulerian formulation with finite elements. East-West J. Numer. Math., 9, 123-156 (2001).

[GiRa79] Girault, V., Raviart, P.-A.: Finite element approximation of the NavierStokes equations. Springer-Verlag, Berlin (1979).

[GPHJP00] Glowinski, R., Pan, T.-W., Hesla, T., Joseph, D., Périaux, J.: A distributed Lagrange multiplier/fictitious domain method for the simulation of flow around moving rigid bodies: application to particulate flow. Comput. Methods Appl. Mech. Engrg., 184, 241-267 (2000).

[GPHJP01] Glowinski, R., Pan, T.-W., Hesla, T. I., Joseph, D.D., Périaux, J.: A fictitious domain approach to the direct numerical simulation of incompressible viscous flow past moving rigid bodies: application to particulate flow. J. Comput. Phys., 169, 363-426 (2001).

[LeTa08] Legendre, G., Takahashi, T.: Convergence of a Lagrange-Galerkin method for a fluid-rigid body system in ALE formulation. M2AN Math. Model. Numer. Anal., 42, 609-644 (2008).

[Ma99] Maury, B.: Direct simulations of 2D fluid-particle flows in biperiodic domains. J. Comput. Phys., 156, 325-351 (1999).

[MoG197] Maury, B., Glowinski, R.: Fluid-particle flow: a symmetric formulation. C. R. Acad. Sci. Paris Sér. I Math., 324, 1079-1084 (1997).

[OsSe88] Osher, S., Sethian, J.A.: Fronts propagating with curvature-dependent speed: algorithms based on Hamilton-Jacobi formulations. J. Comput. Phys., 79, 12-49 (1988).

[Pe02] Peskin, C.S.: The immersed boundary method. Acta Numer., 11, 479517 (2002).

[Pi82] Pironneau, O.: On the transport-diffusion algorithm and its applications to the Navier-Stokes equations. Numer. Math., 38, 309-332 (1982).

[QuVa94] Quarteroni, A., Valli, A.: Numerical approximation of partial differential equations. Springer-Verlag, Berlin (1994). 
[SMSS10a] San Martín, J., Scheid, J.-F., Smaranda, L.: A time discretization scheme of a characteristics method for a fluid-rigid system with discontinuous density. C. R. Math. Acad. Sci. Paris, 348, 935-939 (2010).

[SMSS10b] San Martín, J., Scheid, J.-F., Smaranda, L.: A modified LagrangeGalerkin method for a fluid-rigid system with discontinuous density. Submitted to Numer. Math., (2010).

[SMSTT04] San Martín, J., Scheid, J.-F., Takahashi, T., Tucsnak, M.: Convergence of the Lagrange-Galerkin method for a fluid-rigid system. C. R. Math. Acad. Sci. Paris, 339, 59-64 (2004).

[SMSTT05] San Martín, J., Scheid, J.-F., Takahashi, T., Tucsnak, M.: Convergence of the Lagrange-Galerkin method for the equations modelling the motion of a fluid-rigid system. SIAM J. Numer. Anal., 43, 1536-1571 (2005).

[SMST09] San Martín, J., Smaranda, L., Takahashi, T.: Convergence of a finite element/ALE method for the Stokes equations in a domain depending on time. J. Comput. Appl. Math., 230, 521-545 (2009).

[Su88] Süli, E.: Convergence and nonlinear stability of the Lagrange-Galerkin method for the Navier-Stokes equations. Numer. Math., 53, 459-483 (1988).

[Te83] Temam, R.: Problèmes mathématiques en plasticité. Gauthier-Villars, Montrouge (1983). 